\title{
Continuous nasal positive airway pressure with a mouth leak: effect on nasal mucosal blood flux and nasal geometry
}

\author{
Michael J Hayes, Fiona B McGregor, David N Roberts, Robert C Schroter, \\ Neil B Pride
}

\begin{abstract}
Background - Obstructive sleep apnoea is a common condition. Treatment with nasal continuous positive airway pressure (CPAP), while effective and safe, causes nasal congestion and stuffiness in some patients. The hypothesis that this study aimed to test was that nasal CPAP with a mouth leak and subsequent unidirectional airflow across the nasal mucosa causes an increase in nasal mucosal blood flux and a fall in both nasal volume and minimal cross sectional area. A secondary aim was to study if this could be prevented by humidifying the air inspired with nasal CPAP.
\end{abstract}

Methods - Nasal CPAP was applied to eight normal subjects who kept their mouths open until they had expired 500 litres. The effect of this on nasal mucosal blood flux and nasal geometry was studied with and without humidification using a laser Doppler blood flowmeter and acoustic rhinometer. In addition, nasal mucosal blood flux was measured in four of the eight subjects before and after nasal CPAP with the mouth closed.

Results - Nasal CPAP using room air with the mouth closed did not result in any change in nasal mucosal blood flux; with a mouth leak nasal CPAP using room air was associated with a $65 \%$ increase in nasal mucosal blood flux. There was no change in nasal geometry. Nasal CPAP using humidified air with a mouth leak did not cause any change in nasal mucosal blood flux or nasal geometry.

Conclusion - Nasal CPAP used with an open mouth leads to an increase in nasal mucosal blood flux. This can be prevented by humidifying the air inspired with nasal CPAP.

(Thorax 1995;50:1179-1182)

Keywords: sleep apnoea, nasal mucosal blood flux, laser Doppler flowmetry, acoustic rhinometry, nasal resistance.

Unit, Centre for

Biological and Medical

Systems, Imperial College, London, UK R C Schroter

Correspondence to: Professor N B Pride.

Received 26 October 1994 Returned to authors 20 February 1995 Revised version received 24 July 1995

Accepted for publication 26 July 1995
The obstructive sleep apnoea (OSA) syndrome occurs in up to $4 \%$ of middle aged men and $2 \%$ of middle aged women. ${ }^{1}$ While not all of these patients will need treatment, it has been estimated that, within an average British health district of 250000 , approximately 170 patients would require treatment with nasal continuous positive airway pressure (CPAP). ${ }^{2}$ An under- standing of both the beneficial and adverse effects of nasal CPAP is obviously important in the management of these patients.

Nasal CPAP is an effective treatment for OSA. It is generally well tolerated and safe. Reported side effects include pneumoencephalus in a patient with a cerebrospinal fluid leak, ${ }^{3}$ conjunctivitis, ${ }^{4}$ and epistaxis. ${ }^{5}$ The most commonly reported side effect is of nasal congestion or stuffiness and dryness which was reported in up to $68 \%$ of patients responding to a questionnaire about nasal CPAP. ${ }^{6} \mathrm{~A}$ more recent study has described symptoms of nasal congestion in $20 \%$ of patients. ${ }^{7}$ Parra et al $^{8}$ have shown that a hygroscopic condenser humidifier introduced into the CPAP circuit reduces complaints of nasal dryness.

Patients using nasal CPAP should ideally keep their mouths closed while using the device. During sleep, however, the mouth may fall open. This leads to a large unidirectional flow of air in through the nose and out through the mouth, driven by the positive pressure of the CPAP circuit. Whilst the frequency with which this occurs has not been studied, patients will often describe being woken by such a mouth leak. Attempts to prevent this with chin straps are not always successful.

Strohl et al have shown that cold dry air $\left(0^{\circ} \mathrm{C}\right.$ and $<5 \%$ humidity) inspired through the nose and expired through the mouth at $30 \mathrm{l} / \mathrm{min}$ led to an increase in nasal airway resistance (Raw) after only four minutes. ${ }^{9}$ Conditions in their study were different from those that occur while using nasal CPAP with a mouth leak. Firstly, the flow across the nasal mucosa was generated by ventilation by the subjects rather than nasal CPAP. Secondly, cold dry air rather than room air was used. However, the unidirectional flow of air across the nasal mucosa in their study also occurs in nasal CPAP with a mouth leak. The authors noted that by simply ensuring that the subjects kept their mouths closed and breathed in and out solely through the nose, the changes in nasal Raw could be prevented. They suggested that it was the unidirectional flow of air over the nasal mucosa which led to vascular congestion and nasal obstruction. Richards et al found similar increases in nasal Raw with unidirectional flow across the nasal mucosa. ${ }^{10}$ These investigators used room air and generated the flow with nasal CPAP rather than voluntary respiration. In addition, they found that humidification and warming of the inspired air prevented the change in nasal Raw. Although the time course of the changes in 
nasal Raw reported by these two groups suggests a vascular mechanism, this has not been proven.

We hypothesised that, in subjects treated with nasal CPAP, while the mouth was open the unidirectional airflow across the nasal mucosa would lead to an alteration in nasal mucosal blood flux and nasal geometry that could be detected by laser Doppler flowmetry and acoustic rhinometry, respectively. Furthermore, we wished to establish whether humidification of the inspired air attenuated these effects.

\section{Methods}

Eight subjects (seven men) aged 28-37 years volunteered to enter the trial which was approved by the research ethics committee of the Hammersmith and Queen Charlotte's Special Health Authority. None had a history of atopy, rhinitis, or other nasal problems.

Nasal mucosal blood flux was measured using a laser Doppler flowmeter (MBF3, Moor Instruments, Devon, UK) which uses an $860 \mathrm{~nm}$ wavelength and $3 \mathrm{~mW}$ power laser to assess red blood cell flux in the nasal mucosa. Changes in flux measured with laser Doppler flowmeters have been shown to correlate well with changes in blood flow in various murine organs and human skin. ${ }^{112}$ The instrument was calibrated before each experiment using a standard latex microsphere suspension that gives a linear calibration of the instrument from 0 to 1000 arbitrary units. The recordings were made with a laser Doppler probe positioned 1-3 mm from the inferior turbinate and maintained in position by a customised helmet and brace. Recordings were made every 0.5 seconds for three minutes after a steady baseline had been achieved for 4-5 minutes. Recordings were averaged and results expressed in arbitrary units.

Following the cold air challenge the laser Doppler probe was immediately repositioned and measurements were taken for a further five minutes.

Previous workers ${ }^{910}$ have used posterior rhinomanometry to measure changes in nasal airway resistance. This was not possible with the Doppler probe in place. Nasal volume and minimal cross sectional area were measured with an acoustic rhinometer (G.M. Instruments, Kilwinning, UK). The technique of acoustic rhinometry has been fully described by Hilberg et $a l,{ }^{13}$ but in summary the instrument consists of a spark generator that produces a sound wave. This sound wave is channelled to the anterior nares where an acoustic seal is achieved by the use of a nose piece inserted a few millimetres into the nasal vestibule. Three sizes of nose piece ensure an "airtight" fit. The sound wave is reflected back down the tube by changes in local acoustic impedance resulting from changes in the cross sectional area of the nasal cavity. The reflected sound is detected by a microphone. The data collected are converted to an area distance function by the instrument's software. Cross sectional areas were calculated for a distance of $7 \mathrm{~cm}$ with a spatial resolution of $0.4 \mathrm{~mm}$. Three sound pulses were used for each measurement and the mean minimal cross sectional area and total nasal volume for the first $7 \mathrm{~cm}$ of the nose were recorded for each subject. It was possible to carry out the acoustic rhinometry whilst the laser Doppler probe was in situ by using the opposite nostril. Three acoustic rhinometry readings were taken immediately following the nasal CPAP and the readings averaged.

A standard CPAP circuit (Sullivan APD 2E, ResCare, Abingdon, Oxon, UK) set to deliver $\mathrm{CPAP}$ at $10 \mathrm{~cm} \mathrm{H}_{2} \mathrm{O}$ pressure through a nasal mask was used for the nasal CPAP. Subjects were studied during the day, while awake, and in the seated position.

The effect of nasal CPAP with a mouth leak was studied in all eight subjects. In addition, the effect of nasal CPAP without a mouth leak was studied in four of the eight subjects. To assess the effect of nasal CPAP with a mouth leak subjects were asked to keep their mouth open while the nasal CPAP was applied, breathing in through their nose and out through their mouth. This arrangement generated a large flow of air across the nasal mucosa but meant it was not possible to measure end tidal carbon dioxide or minute ventilation. Airflow out of the mouth was measured with a Wright respirometer (Ferraris Medical, London, UK). CPAP was stopped when 500 litres had been expired through the mouth. Typically this took around 10 minutes, giving a flow rate of approximately $50 \mathrm{l} / \mathrm{min}$. Measurements of nasal mucosal blood flux and nasal geometry were made both before and immediately after the nasal CPAP.

On a second day the entire procedure was repeated with the addition of a humidifier (Sullivan HC100, ResCare, Abingdon, Oxon, UK) to the CPAP circuit. The humidifier was inserted in the CPAP circuit between the CPAP machine and the subject. Air from the CPAP machine was passed over a heated water bath. This both warmed and humidified the air reaching the subject. The humidifier was set to run at maximum during the CPAP application.

When CPAP was used without humidification, measurements of ambient temperature and humidity were made with a thermometer and hygrometer. The temperature and humidity of the air delivered by the CPAP with the humidifier were measured in the circuit at the junction with the nasal mask with a humidity and temperature indicator (HMI 37, Vaisala, Finland).

To assess whether nasal CPAP alone without a mouth leak could have had an effect on nasal mucosal blood flux, measurements of nasal mucosal blood flux before and after nasal CPAP with the mouth closed were made in four of the subjects. Nasal CPAP at $10 \mathrm{~cm} \mathrm{H}_{2} \mathrm{O}$ pressure was applied using room air for 10 minutes which was the average time the nasal CPAP was applied in the mouth leak studies.

Results before and after CPAP were compared using a paired Student's $t$ test. Significance was defined as a $p$ value of $<0.05$. 
Comparison of nasal volume, minimal cross sectional area, and mucosal blood flux before and after nasal CPAP with mouth open in room and humidified air

\begin{tabular}{|c|c|c|c|c|c|c|c|c|c|c|c|c|}
\hline & \multicolumn{4}{|c|}{$\begin{array}{l}\text { Nasal volume } \\
\left(\mathrm{cm}^{3}\right)\end{array}$} & \multicolumn{4}{|c|}{$\begin{array}{l}\text { Nasal minimal cross sectional area } \\
\left(\mathrm{cm}^{2}\right)\end{array}$} & \multicolumn{4}{|c|}{$\begin{array}{l}\text { Nasal mucosal blood flux } \\
\text { (arbitrary units) }\end{array}$} \\
\hline & \multicolumn{2}{|c|}{ Room air } & \multicolumn{2}{|c|}{ Humidified } & \multicolumn{2}{|c|}{ Room air } & \multicolumn{2}{|c|}{ Humidified } & \multicolumn{2}{|c|}{ Room air } & \multicolumn{2}{|c|}{ Humidified } \\
\hline & Before & After & Before & After & Before & After & Before & After & Before & After & Before & After \\
\hline $\begin{array}{l}\text { Mean } \\
\text { SD } \\
\text { p }\end{array}$ & $\begin{array}{r}18 \cdot 22 \\
6 \cdot 49\end{array}$ & 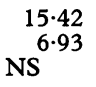 & $\begin{array}{r}14 \cdot 84 \\
4 \cdot 11\end{array}$ & 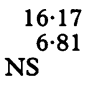 & $\begin{array}{l}0 \cdot 94 \\
0 \cdot 26\end{array}$ & $\begin{array}{l}0.82 \\
0 \cdot 30 \\
\text { NS }\end{array}$ & $\begin{array}{l}0.91 \\
0.28\end{array}$ & $\begin{array}{l}0 \cdot 86 \\
0 \cdot 23 \\
\text { NS }\end{array}$ & $\begin{array}{l}430 \\
105\end{array}$ & $\begin{array}{r}688 \\
109 \\
<0.0001\end{array}$ & $\begin{array}{l}376 \\
113\end{array}$ & $\begin{array}{l}407 \\
189 \\
\text { NS }\end{array}$ \\
\hline
\end{tabular}

\section{Results}

Room air temperature and humidity at the time of the non-humidified CPAP averaged $20^{\circ} \mathrm{C}$ and $51 \%$, respectively. Temperature and humidity of the inspired air using the humidifier averaged $29^{\circ} \mathrm{C}$ and $70 \%$.

\section{BASELINE MEASUREMENTS AND VARIABILITY (table)}

Baseline measurements of nasal mucosal blood flux and nasal geometry were made on day 1 before room air nasal CPAP and on day 2 before humidified nasal CPAP. The baseline measurements of blood flux and minimal cross sectional area were not significantly different on the two days, but nasal volume was significantly less on day $2(\mathrm{p}<0.05)$. Mean intra-individual coefficients of variation for the two baseline measurements of nasal volume, minimal cross sectional area, and mucosal flux were $14 \%$, $11 \%$ and $17 \%$, respectively.

NASAL CPAP WITHOUT A MOUTH LEAK

In the four subjects studied, mean (SD) nasal mucosal blood flux was 382 (102) arbitrary units before the nasal CPAP and 347 (127) arbitrary units after the nasal CPAP. The difference was not significant (figure).

NASAL CPAP WITH A MOUTH LEAK

Every subject had an increase in nasal mucosal blood flux after nasal CPAP using room air

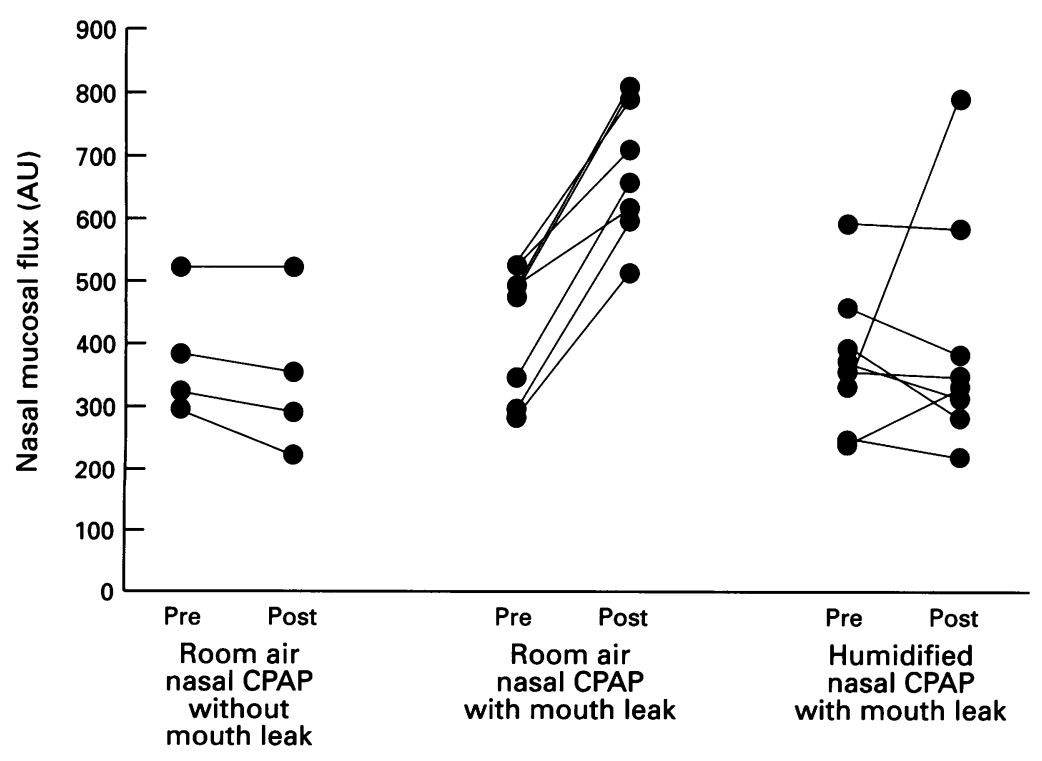

Nasal mucosal flux with and without mouth leak before and after nasal CPAP. (figure). The mean increase in blood flux was $65 \%(\mathrm{p}<0.0001)$ (table) compared with a coefficient of variation of $17 \%$ in baseline measurement of blood flux. There was a small non-significant change in nasal volume.

Nasal mucosal blood flux and nasal geometry after humidified nasal CPAP were not significantly different from measurements taken immediately before humidified nasal CPAP. Nasal mucosal blood flux increased by only $8 \%$ compared with an increase of $65 \%$ with room air nasal CPAP.

\section{Discussion}

Patients being treated with nasal CPAP during sleep may open their mouths which leads to a leak of air out the mouth and a large unidirectional flow of air across the nasal mucosa. Although patients often describe this phenomenon it has not been studied in any great detail. In this study 500 litres were passed through the nose. Although this seems a large volume it took on average only 10 minutes. Patients using nasal CPAP have the potential to develop mouth leaks over the entire night.

This study was carried out in normal subjects while awake and sitting. At least in these circumstances nasal CPAP causes an increase in nasal mucosal blood flux. The increase occurred in all subjects and was significant for the group as a whole. The present study did not investigate the time course of this change, but in similar studies nasal Raw starts returning towards baseline within minutes of stopping the stimulus. ${ }^{910}$

Humidification and warming of the inspired air with a humidifier suitable for home use prevents the increase in nasal mucosal blood flux. The fact that the increase in blood flux could be prevented in this way suggests that it is the cooling or drying effect of the airflow which is responsible for the change.

Although subjects were instructed to breathe normally throughout the experiments, it is possible that they could have hyperventilated. Hyperventilation is thought to increase nasal resistance via an effect on levels of blood carbon dioxide tension. ${ }^{14} 15$ The arrangement of the circuit used made it impossible to measure end tidal carbon dioxide so we were unable to exclude this possibility. However, the fact that humidification prevented the change in blood flux suggests that the subjects did not hyperventilate.

It has been shown that, when the nose is exposed to cooling and drying, the nasal mucosa releases vasoactive amines and 
leukotrienes. ${ }^{1617}$ These mediators increase both superficial mucosal blood flux and deeper capacitance vessel engorgement. Engorgement of the capacitance vessels leads to a rise in nasal resistance.

We had expected to see a fall in nasal volume and cross sectional area in combination with the increase in nasal mucosal blood flux. Richards et al, using a very similar circuit (nasal CPAP with open mouth), were able to show an increase in nasal airway resistance using posterior rhinomanometry. ${ }^{10} \mathrm{We}$ were unable, however, to detect any significant change using acoustic rhinometry. Acoustic rhinometry and posterior rhinomanometry have been compared in the assessment of nasal changes during nasal histamine challenges. ${ }^{18}$ Whilst the two methods correlated quite well under these circumstances, the delivered doses of histamine in these challenges were greatly in excess of the physiological concentrations found when the nose is stimulated by cold dry air. ${ }^{16}$ Consequently, it could be postulated that the nasal volume changes caused by the release of inflammatory mediators during the present study were too small to be detected by acoustic rhinometry, and that posterior rhinomanometry is a more sensitive technique in this circumstance.

In conclusion, we have shown that even a brief period of nasal CPAP with an open mouth leads to an increase in nasal mucosal blood flux. This effect was not seen when nasal CPAP was applied and the mouth was kept closed. Even with a mouth leak the change in nasal mucosal blood flux could be prevented by warming and humidifying the inspired air. Extrapolation of these results to the sleeping state, older subjects, or subjects with nasal pathology needs to be approached with caution. However, the results of our study may have implications for the management of patients being treated with nasal CPAP.
The authors would like to thank Dr Glen Richards for his advice and assistance. Two of the authors (FMcG and DR) were supported in this work by a grant from the Health and Safety Executive.

1 Young T, Palta M, Dempsey J, Skatrud J, Weber S, Badr $S$. The occurrence of sleep-disordered breathing among middle aged adults. $N$ Engl $¥ \mathrm{Med} 1993 ; 328: 1230-5$.

2 Semple SIG (chairman). Sleep apnoea and related conditions. A report of a working party of the Royal College of Physicians. London: The Royal College of Physicians of London, 1993:19.

3 Janjour N, Wilson P. Pneumoencephalus associated with nasal CPAP in a patient with sleep apnoea. Chest 1989 , 96:1425-6.

4 Strauffer J, Fayter N, MacLurg B. Conjunctivitis from nasa CPAP apparatus (letter to editor). Chest 1984;86:802.

5 Strumpf DA, Harrop P, Dobbin J, Millman R. Massive epistaxis from nasal CPAP therapy. Chest 1989;95:1141.

6 Sanders M, Gruendl C, Rogers R. Patient compliance with Sanders M, Gruendl C, Rogers R. Patient compliance with
nasal CPAP therapy for sleep apnoea. Chest 1986;90: 330-3.

7 Engelman H, Martin S, Douglas N. Compliance with nasal CPAP therapy in patients with the sleep apnoea/hypopnoea syndrome. Thorax 1994;49:263-6.

8 Parra O, Klamburg J, Xirgu J, Abad J, Sala H, Tomasa A, et al. Hygroscopic condenser humidifier as a solution to nasal dryness due to nasal CPAP treatment for obstructive sleep apnoea syndrome. Sleep 1991;14:166-8.

9 Strohl K, Arnold J, Decker M, Hoekje P, McFadden E. Nasal flow resistive responses to challenge with cold dry air. $\mathcal{F}$ Appl Physiol 1992;72:1243-6.

10 Richards $G$, Cistulli $P$, Unger R, Berrthon-Jones M, Sullivan C. Mouth leak with nasal CPAP increases nasal airway resistance (abstract). Aust NZ $\mathcal{f}$ Med 1994;24:480.

11 Smits GJ, Roman RJ, Lombard JH. Evaluation of laserDoppler flowmetry as a measure of tissue blood flow. $\mathcal{f}$ Appl Physiol 1986;61:666-72.

12 Stern MD, Lappe DL, Bowen PD, Chimosky JE, Holloway GJ, Keiser HR, et al. Continuous measurement of tissue blood flow by laser-Doppler spectroscopy. Am f Physiol 1977;232: H441-8.

13 Hilberg O, Jackson A, Swift D, Pederson O. Acoustic rhinometry: evaluation of nasal cavity geometry by acoustic reflection. $\mathcal{F} A p p l$ Physiol 1989;66:295-303.

14 Hasegawa M, Kern EB. The effect of breath holding, hyperventilation, and exercise on nasal resistance. Rhinology 1978;16:243-9.

15 Dallimore NS, Eccles R. Changes in human nasal resistance associated with exercise, hyperventilation and rebreathing. Acta Otolaryngol Stockh 1977;84:416-21.

16 Togias A, Naclerio R, Proud D, Fish J, Adkinson NJ, KageySobotka A, et al. Nasal challenge with cold dry air results in release of inflammath mediators. Possible mast cell involvement. $\mathcal{F}$ Clin Invest 1985;76:1375-81.

17 Togias A, Naclerio R, Peters S, Nimmagadda I, Proud D, Kagey-Sobotka A, et al. Local generation of sulfidopeptide leukotrienes upon nasal provocation with cold, dry air. Am Rev Respir Dis 1986;133:1133-7.

18 Austin C, Foreman J. Acoustic rhinometry compared with posterior rhinomanometry in the measurement of histamine and bradykinin induced changes in nasal airway patency. Br f Clin Pharmacol 1994;37:33-7. 